\title{
Anisotropy evolution of magnetic field fluctuation through the bow shock
}

\author{
Y. Narita ${ }^{1}$ and K.-H. Glassmeier ${ }^{1,2}$ \\ ${ }^{1}$ Institut für Geophysik und extraterrestrische Physik, Technische Universität Braunschweig, \\ Mendelssohnstraße 3, D-38106 Braunschweig, Germany \\ ${ }^{2}$ Max-Planck-Institut für Sonnensystemforschung, Max-Planck-Straße 2, D-37191 Katlenburg-Lindau, Germany
}

(Received September 30, 2009; Revised January 29, 2010; Accepted February 1, 2010; Online published February 22, 2010)

\begin{abstract}
Measurement of energy distributions in the wave vector domain reveals how anisotropy of turbulent magnetic field fluctuations evolves as the solar wind encounters the terrestrial bow shock and the magnetosphere. While fluctuations in the solar wind, the magnetosheath, and the magnetospheric cusp regions are characterized by the perpendicular wave vector geometry to the mean magnetic field direction, that in the foreshock region is characterized by the parallel wave vector geometry. Linear and nonlinear plasma processes are discussed for the anisotropy evolution.
\end{abstract}

Key words: Wave-vector spectra, magnetic field, bow shock, multi-point measurements.

Many in-situ spacecraft observations suggest that shock waves in the interplanetary space such as planetary bow shocks and traveling shocks in the co-rotating interaction regions are often accompanied by turbulent fluctuations, and furthermore, there are indications that interstellar shocks or supernova remnants may be associated with turbulence (Hester et al., 1994; Spitler and Spangler, 2005). How turbulence evolves as it encounters the shock wave in a collisionless plasma is an interesting problem and of particular importance in space physics and astrophysics. Earth's bow shock, a standing shock wave located at about 20 Earth radii in front of the Earth, serves as an ideal, natural laboratory for studying turbulence evolution across the shock. Many spacecraft visited the Earth's bow shock and observed various kinds of electrostatic and electromagnetic fluctuations near the shock, e.g., Shin et al. (2007). Spatial properties of waves or turbulence in the surroundings of the bow shock can be extensively studied by the Cluster mission (Escoubet et al., 2001), as it provides four-point measurements in the near-Earth space.

Here we present a measurement of energy distributions of magnetic field fluctuations in the wave vector domain using the Cluster fluxgate magnetometer data (Balogh et al., 2001). We use the concept of two distinct fluctuation geometries to study anisotropy: parallel and perpendicular wave vector geometries (Fig. 1). The idea of the two fluctuation geometries is motivated by long-standing questions about the nature of symmetries of plasma turbulence, viz., whether wave vectors in plasma turbulence prefer parallel or perpendicular directions to the mean magnetic field (Matthaeus et al., 1990; Carbone et al., 1995).

We investigate the magnetic field data for two Cluster or-

Copy right(c) The Society of Geomagnetism and Earth, Planetary and Space Sciences (SGEPSS); The Seismological Society of Japan; The Volcanological Society of Japan; The Geodetic Society of Japan; The Japanese Society for Planetary Sciences; TERRAPUB.

doi:10.5047/eps.2010.02.001 bits of bow shock crossings during the mission phase with about $100 \mathrm{~km}$ mean spacecraft separation. The orbit of the first crossing (orbit A) encountered (1) the solar wind, (2) the foreshock, and (3) the magnetosheath. The orbit for the second crossing (orbit B) encountered (4) the solar wind, (5) the magnetosheath, and (6) the magnetospheric cusp region. While the first orbit represents a crossing of the quasiparallel shock (angle between the shock normal and the upstream magnetic field $30.5 \mathrm{deg}$ ), the second one represents a crossing of the quasi-perpendicular shock (angle $75.7 \mathrm{deg}$ ). Figure 2 displays the observed magnetic field magnitude for the two shock crossings. The orbit $\mathrm{A}$ is inbound and encountered the shock crossing at about 1600 UT and the magnetopause crossing at about 2130 UT. We use the interval (1) Feb. 11, 2002, 1730-2030 UT for the solar wind, (2) Feb. 12, 2002, 0630-1230 UT for the foreshock, and (3) 1615-2100 UT for the magnetosheath. The orbit B is outbound. After exiting the nightside magnetosphere just before $0900 \mathrm{UT}$, the spacecraft re-entered the magnetosphere on the dayside at about 1000 UT and encountered the dayside magnetopause and the shock at about 1015 UT and 1350 UT, respectively. We use the intervals (4) Mar. 4, 2002, 1415-1615 UT for the solar wind, (5) 1015-1330 UT for the magnetosheath, and (6) 0900-0945 UT for the cusp. The cusp region is an extended part of the magnetosheath where the flow becomes trapped and stagnant, and is known to be in a turbulent state, e.g., Pilipenko et al. (2008). Therefore it is an interesting question how the wave vector anisotropy develops when the solar wind enters the magnetosheath and further encounters the cusp region.

With Cluster data it is possible to measure the cross spectral density matrix of magnetic field fluctuations directly in the three-dimensional wave vector domain without using Taylor's hypothesis. From this matrix we obtain the magnetic energy distribution in the wave vector domain. The distribution is measured up to the Nyquist wave number $k_{\max }=3.0 \times 10^{-2} \mathrm{rad} / \mathrm{km}$ that is deter- 


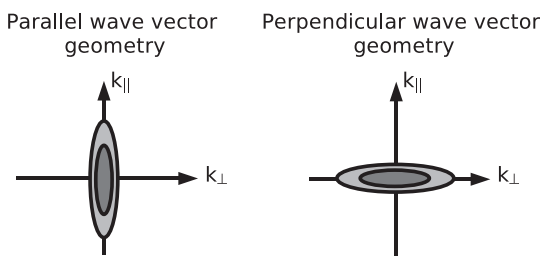

Fig. 1. Contours of energy distributions for two fluctuation geometries.
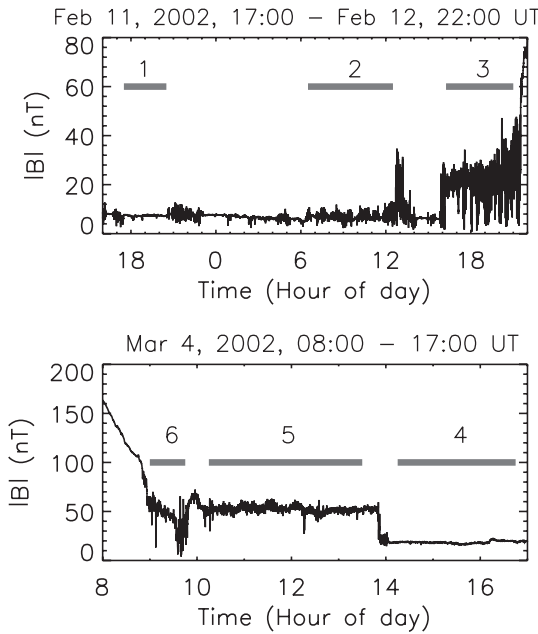

Fig. 2. Time series plots of the magnetic field magnitude observed by Cluster-1 for orbit A (top, quasi-parallel shock crossing) and B (bottom, quasi-perpendicular shock crossing). Intervals $1, \cdots, 6$ represent the solar wind, the foreshock, the magnetosheath, the solar wind, the magnetosheath, and the cusp region that are used for the analysis, respectively.

mined by the spacecraft separation distance (cf. wave numbers of the ion inertial length are about $0.008,0.009,0.017$, $0.013,0.026$, and $0.014 \mathrm{rad} / \mathrm{km}$ for the region 1 to 6 , respectively). The wave telescope technique (or k-filtering technique) is used to determine the fluctuation energy in the frequency and wave vector domain. This technique was developed particularly for analyzing multi-spacecraft data (Pinçon and Lefeuvre, 1991; Motschmann et al., 1996; Glassmeier et al., 2001). The distribution is then integrated over spacecraft-frequency up to the Doppler limit $k_{\max } V_{\text {flow }}$, where the flow speed $V_{\text {flow }}$ is obtained by the measurement of the ion bulk flow using electrostatic analyzer CIS-HIA on board Cluster (Rème et al., 2001). Figure 3 displays the energy distributions averaged over the directions around the mean magnetic field for the six analyzed regions. The distributions are furthermore reduced to one-dimensional energy spectra for the two fluctuation geometries by summing over the parallel or the perpendicular wave numbers, respectively, and then the spectra are compared at the same wave numbers by setting $k_{\|}=k_{\perp}$ to measure the wave vector anisotropy. Figure 4 displays the ratios of the energy for the parallel to the perpendicular wave vector geometry. A positive trend of the ratio in the wave number domain represents the dominance of the parallel wave vector geometry, and vice versa. Figure 4 also exhibits an error estimate for the determined energy. The error in the energy determina-
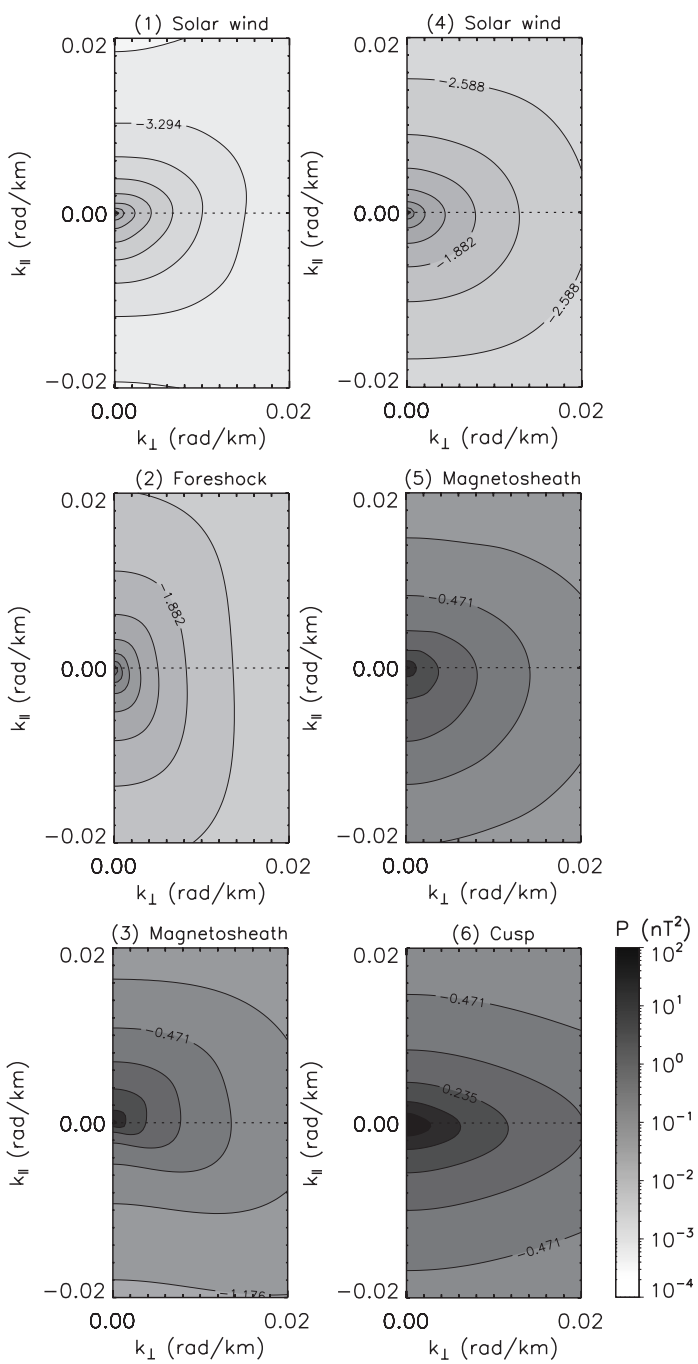

Fig. 3. Energy distribution of magnetic field fluctuation in the wave vector domain for six different regions across the bow shock. The distribution is integrated over spacecraft-frame frequency and averaged over the directions around the mean magnetic field. The numbers at the contour lines are the logarithm (with base 10) of the determined energy.

tion comes primarily from the motion of the spacecraft and the change of spacecraft distance during the observations, which is on average about $15 \%$ to the determined energy.

In the solar wind (region 1 and 4) the fluctuation energy is the smallest for the both shock crossings. In region 1 the distribution is extended in the perpendicular direction to the mean magnetic field and is elliptically shaped on an intermediate scale (about $0.010 \mathrm{rad} / \mathrm{km}$ ), while it is moderately rectangular with dominant extension in the perpendicular direction at larger wave numbers (about $0.020 \mathrm{rad} / \mathrm{km}$ ). In region 4 the distribution is also extended in the perpendicular direction. The ratio of the two reduced spectra exhibits a negative trend down to values $0.8-0.9$ toward larger wave numbers in the both cases, reflecting the dominance of the perpendicular wave vector geometry. These results justify the picture of two-dimensional turbulence in the solar wind (Matthaeus et al., 1990; Carbone et al., 1995). In the foreshock region (region 2) the fluctuation energy becomes larger than that of the solar wind by factor about 10 . In contrast to the solar wind, the energy distribution is extended in 
(A) Quasi-parallel shock crossing

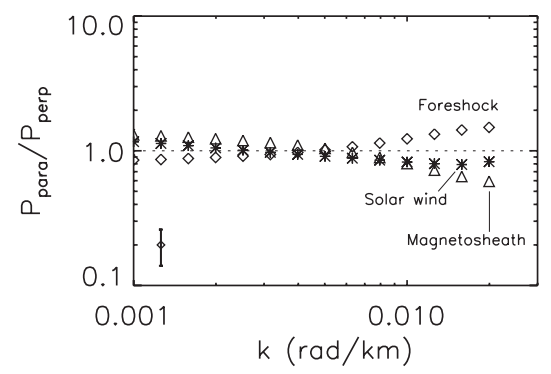

(B) Quasi-perpendicular shock crossing

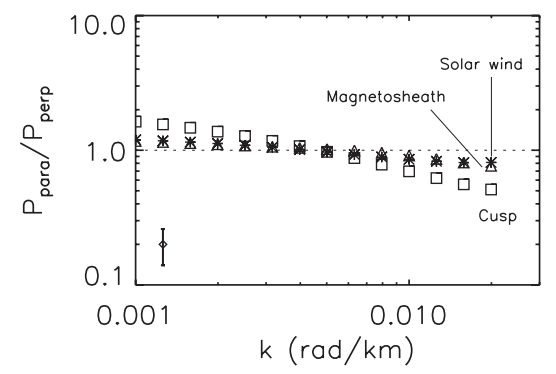

Fig. 4. Ratio of the reduced energy spectra for the parallel wave vector geometry to that for the perpendicular wave vector geometry compared at the same wave numbers by setting $k_{\|}=k_{\perp}$.

the parallel direction to the mean magnetic field and represents the dominance of the parallel wave vector geometry, which is reflected in the anisotropy ratio as a positive trend up to the ratio 1.5 .

In the magnetosheath (region 3 and 5) the fluctuation energy is further enhanced from the foreshock by factor about 10 (region 3) and from the solar wind by factor about 50 (region 5). The distribution in region 3 exhibits an asymmetric feature between the parallel and the anti-parallel directions as well as anisotropy between the parallel and the perpendicular directions. The ratio of the two reduced spectra exhibits a negative trend down to 0.6 and suggests the dominance of the perpendicular wave vector geometry. Anisotropy preferring the perpendicular wave vector geometry is stronger than that of the solar wind (region 1). In region 5 the fluctuation energy is enhanced from the solar wind across the shock while the distribution maintains the extended structure in the perpendicular direction. Also, there is a moderate asymmetry between the parallel and the anti-parallel directions. The ratio of the reduced spectra exhibits a negative trend, preferring the perpendicular wave vector geometry, and the anisotropy ratio curve is very similar to that of the solar wind (region 4).

In the cusp region (region 6) the fluctuation energy is enhanced from the magnetosheath and the energy distribution is further extended in the perpendicular direction. The anisotropy ratio curve exhibits a negative trend 0.5 , which may suggest that cusp turbulence inherits the properties of the magnetosheath fluctuations.

There are both new results and confirmation of previous results in our analysis. The anisotropies between parallel and perpendicular directions to the mean magnetic field support the results obtained by earlier spacecraft measurements. The measurements before Cluster were limited to one or at most two-point measurements (Le and Russell, 1990; Matthaeus et al., 1990; Carbone et al., 1995) and it was not known about how general those results are in the 3-D space. Our measurements with Cluster data not only justify the earlier results of the anisotropic features but also provide the quantitative estimate of the anisotropies in the 3-D space, which is new. With Cluster data the anisotropies and the asymmetries of the energy distribution are visualized for the first time. Here, it should be noted that our analysis is performed in the spacecraft frame and the results are subject to the Doppler shift due to the presence of the mean flow. Doppler shift correction will be needed to verify our results.

One of likely sources for the anisotropies of turbulent fluctuations is plasma instabilities. In the foreshock, electron-beam and ion-beam modes are the most likely instabilities, because the bow shock is a source of heated electrons and reflected ions. In the frequency range of concern to us (up to about ion gyro-frequency), the most likely mode to grow is the electromagnetic ion/ion righthand resonant instability. This has been confirmed observationally by Watanabe and Terasawa (1984) and Fuselier et al. (1986) using single spacecraft methods and recently by multi-spacecraft methods of Cluster (Narita et al., 2003; Narita and Glassmeier, 2005). Linear kinetic theory (Gary, 1993) shows that the instability has maximum growth in the parallel and anti-parallel directions to the mean magnetic field, so that enhanced fluctuations from this instability should have properties of the parallel wave vector geometry. Magnetosheath plasma shows the consequences of magnetic compression and heating at the shock, so that the primary characteristic of proton distributions in this regime is a strong temperature anisotropy. This anisotropy leads to the growth of both electromagnetic ion cyclotron and mirror-mode fluctuations with the mirror instability often dominating the high- $\beta$ plasmas near and downstream of the shock. The mirror instability has maximum growth at directions strongly oblique to the mean magnetic field, so that enhanced fluctuations from this growing mode should have properties of the perpendicular wave vector geometry. Anisotropy in the cusp region can also be qualitatively explained by the mirror mode fluctuations. Luhmann et al. (1986) argued that one of the major sources of fluctuations downstream of the quasi-parallel shock is the foreshock activity, but our result suggests that the property of the foreshock fluctuations is lost across the shock. Our result is consistent with the statistical analysis of Cluster data that the foreshock fluctuation property is lost in the magnetosheath (Narita et al., 2006). It is also worthwhile to note that the perpendicular wave vector geometry may be interpreted not only as the mirror mode but also as quasi-two-dimensional turbulence. Distinguishing these two fluctuation types will require polarization or helicity analysis combined with the wave telescope technique.

It was also discovered in our results that the fluctuation energy is on the whole evenly distributed between parallel and anti-parallel directions to the mean magnetic field. In particular, the energy distribution in the foreshock region is almost symmetric between these two directions, while the analysis of dispersion relations gives a preferred direc- 
tion for wave propagation (Narita et al., 2003; Narita and Glassmeier, 2005). The symmetric distribution may be a sign that nonlinear wave-wave interactions, such as decay instability, are operating so that the fluctuation energy becomes distributed in both parallel and anti-parallel directions. But strictly speaking, the energy distributions exhibit asymmetries and distortions, particularly in the two magnetosheath regions. Possible causes of the asymmetries would be: waves propagating in a preferred direction; Doppler effect; and spatial aliasing (Sahraoui et al., 2003; Narita and Glassmeier, 2009). We also note that even though we observe fluctuations downstream of the bow shock with a particular magnetic field geometry (or shock angle), the plasma may be also affected by other portions of the shock (having different shock angles) which are magnetically connected to the spacecraft positions, as discussed by Feldman et al. (1983).

Another process which may be relevant is wave amplification across the shock. Fluctuations in the solar wind may be amplified at the bow shock independently of any instabilities. The interaction of the magnetohydrodynamic waves with the shock wave was analytically studied by McKenzie and Westphal (1969, 1970) and McKenzie (1970). Their analysis suggests that the magnetic field amplitude of an Alfvén wave incident in the shock-upstream region is enhanced by a factor of unity or three, depending on the sense of wave propagation in the upstream and downstream region with respect to the shock normal direction and that the amplification of a fast magnetosonic wave is about a factor of four. Therefore a naive estimate gives the jump of the spectral power by factors 10-20 across the shock, as energy is proportional to the squared amplitude of fluctuation. We obtain in our measurement the jump of the energy by factor about 10 from the foreshock to the magnetosheath across the quasi-parallel shock, and about 50 across the quasi-perpendicular shock. Probably there are a variety of mechanisms that contribute to the amplification across the shock such as wave mode conversion and wave reflection at the shock or at the magnetopause. In this analysis it is difficult to distinguish between plasma instabilities and shock amplification effects because only the total energy is used in the analysis. The polarization or helicity analysis will verify our results and help us to distinguish these two effects, as such an analysis can determine energy spectra for different fluctuation components and for different wave vectors.

Acknowledgments. This work was financially supported by Bundesministerium für Wirtschaft und Technologie and Deutsches Zentrum für Luft- und Raumfahrt, Germany, under contract 50 OC 0901. We thank H. Rème and I. Dandouras for providing the ion data of Cluster, and S. P. Gary for discussion.

\section{References}

Balogh, A., C. M. Carr, M. H. Acuña, M. W. Dunlop, T. J. Beek, P. Brown, K.-H. Fornaçon, E. Georgescu, K.-H. Glassmeier, J. Harris, G. Musmann, T. Oddy, and K. Schwingenschuh, The Cluster magnetic field investigation: overview of in-flight performance and initial results, Ann. Geophys., 19, 1207-1217, 2001.

Carbone, V., F. Malara, and P. Veltri, A model for the three-dimensional magnetic field correlation spectra of low-frequency solar wind fluctuations during Alfvénic periods, J. Geophys. Res., 100, 1763-1778, 1995.

Escoubet, C. P., M. Fehringer, and M. Goldstein, The Cluster mission, Ann. Geophys., 19, 1197-1200, 2001.
Feldman, W. C., R. C. Anderson, S. J. Bame, S. P. Gary, J. T. Gosling, D. J. McComas, and M. F. Thomsen, Electron velocity distributions near the Earth's bow shock, J. Geophys. Res., 88, 96-110, 1983.

Fuselier, S. A., M. F. Thomsen, J. T. Gosling, S. J. Bame, and C. T. Russell, Gyrating and intermediate ion distributions upstream from the earth's bow shock, J. Geophys. Res., 91, 91-99, 1986.

Gary, S. P., Theory of Space Plasma Microinstabilities, pp. 123-169, Cambridge Univ. Press, Cambridge, U.K., 1993.

Glassmeier, K.-H., U. Motschmann, M. Dunlop, A. Balogh, M. H Acuña, C. Carr, G. Musmann, K.-H. Fornaçon, K. Schweda, J. Vogt, E. Georgescu, and S. Buchert, Cluster as a wave telescope-first results from the fluxgate magnetometer, Ann. Geophys., 19, 1439-1447, 2001 (correction 21, 1071, 2003).

Hester, J. J., J. C. Raymond, and W. P. Blair, Astrophys. J., 420, 721-745, 1994.

Le, G. and C. T. Russell, Study of the coherence length of ULF waves in the Earth's foreshock, J. Geophys. Res., 95, 10,703-10,706, 1990.

Luhmann, J. G., C. T. Russell, and R. C. Elphic, J. Geophys. Res., 91, $1711-1715,1986$

Matthaeus, W. H., M. L. Goldstein, and D. A. Roberts, Evidence for the presence of quasi-two-dimensional nearly incompressible fluctuations in the solar wind, J. Geophys. Res., 95, 20,673-20,683, 1990.

McKenzie, J. F., Hydromagnetic wave interaction with the magnetopause and the bow shock, Planet. Space Sci., 18, 1-23, 1970.

McKenzie, J. F. and K. O. Westphal, Transmission of Alfvén waves through the Earth's bow shock, Planet. Space Sci., 17, 1029-1037, 1969.

McKenzie, J. F. and K. O. Westphal, Interaction of hydromagnetic waves with hydromagnetic shocks, Phys. Fluids., 13, 630-640, 1970.

Motschmann, U., T. I. Woodward, K. H. Glassmeier, D. J. Southwood, and J. L. Pinçon, Wavelength and direction filtering by magnetic measurements at satellite arrays: Generalized minimum variance analysis, J. Geophys. Res., 101, 4961-4966, 1996.

Narita, Y. and K.-H. Glassmeier, Dispersion analysis of low-frequency waves through the terrestrial bow shock, J. Geophys. Res., 110, A12215, doi:10.1029/2005JA011256, 2005.

Narita, Y. and K.-H. Glassmeier, Spatial aliasing and distortion of energy distribution in the wave vector domain under multi-spacecraft measurements, Ann. Geophys., 27, 3031-3042, 2009.

Narita, Y., K.-H. Glassmeier, S. Schäfer, U. Motschmann, K. Sauer, I. Dandouras, K.-H. Fornaçon, E. Georgescu, and H. Rème, Dispersion analysis of ULF waves in the foreshock using cluster data and the wave telescope technique, Geophys. Res. Lett., 30, SSC 43-1, doi:10.1029/2003GL017432, 2003.

Narita, Y., K.-H. Glassmeier, K.-H. Fornaçon, I. Richter, S. Schäfer, U. Motschmann, I. Dandouras, H. Rème, and E. Georgescu, Low frequency wave characteristics in the upstream and downstream regime of the terrestrial bow shock, J. Geophys. Res., 111, A01203, doi:10.1029/2005JA011231, 2006.

Pilipenko, V., E. Fedorov, and M. J. Engebretson, Interaction of Alfven waves with a turbulent layer, Earth Planets Space, 60, 949-960, 2008.

Pinçon, J. L. and F. Lefeuvre, Local characterization of homogeneous turbulence in a space plasma from simultaneous measurements of field components at several points in space, J. Geophys. Res., 96, 1789-1802, 1991.

Rème, H., C. Aoustin, J. M. Bosqued, I. Dandouras et al., First multispacecraft ion measurements in and near the Earth's magnetosphere with the identical Cluster ion spectrometry (CIS) experiment, Ann. Geophys., 19, 1303-1354, 2001.

Sahraoui, F., J. L. Pinçon, G. Belmont, L. Rezeau, N. Cornilleau-Wehrlin, P. Robert, L. Mellul, J. M. Bosqued, A. Balogh, P. Canu, and G. Chanteur, ULF wave identification in the magnetosheath: The k-filtering technique applied to Cluster II data, J. Geophys. Res., 108, SMP 11, CiteID 1335, doi:10.1029/2002JA009587, 2003 (correction in $\mathbf{1 0 9}$ A04222, doi10.1029/2004JA010469, 2004).

Shin, K., H. Kojima, H. Matsumoto, and T. Mukai, Electrostatic quasimonochromatic waves in the downstream region of the Earth's bow shock based on Geotail observations, Earth Planets Space, 59, 107-112, 2007.

Spitler, L. G. and S. R. Spangler, Limits on enhanced radio wave scattering by supernova remnants, Astrophys. J., 632, 932-940, 2005.

Watanabe, Y. and T. Terasawa, On the excitation mechanism of the lowfrequency upstream waves, J. Geophys. Res., 89, 6623-6630, 1984.

Y. Narita (e-mail: y.narita@tu-bs.de) and K.-H. Glassmeier 